\title{
Antigenicity of cystatin-binding proteins from parasitic protozoan Detection by a proteinase inhibitor based capture immunoassay (PINC-ELISA)
}

\author{
Julio Scharfstein ${ }^{a, *}$, Magnus Abrahamson ${ }^{b}$, Clarissa B. Palatnik de Souza ${ }^{c}$, \\ Aldina Barral ${ }^{\mathrm{d}}$, Ian Victor Silva ${ }^{\mathrm{a}}$

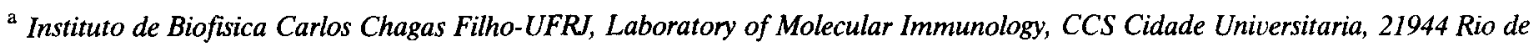 \\ Janeiro, Brazil \\ ${ }^{\mathrm{b}}$ Department of Clinical Chemistry, University of Lund, Lund, Sweden \\ ${ }^{c}$ Instituto de Microbiologia, UFRJ, Rio de Janeiro, Brazil \\ ' Serviço de Imunologia do Hospital Universitário Edgard Santos, Universidade Federal da Bahia, Universidade Federal da Bahia, \\ Salvador, Brazil
}

Received 26 July 1994; revised 17 January 1995; accepted 17 January 1995

\begin{abstract}
A novel immunoassay (PINC-ELISA) was designed using proteinase inhibitors of the cystatin superfamily (PINC) in the solid phase, to promote the selective capture of cysteine proteinases. The method was applied in the identification of papain-like antigens from parasitic protozoa. PINC of human origin, namely recombinant cystatin C ( $\mathrm{r}$-cystatin $\mathrm{C}$ ) or low molecular weight kininogen were used in the assays to adsorb proteases contained in cell lysates from various trypanosomatids. The PINC-ELISA was at first optimized with the major cysteine proteinase from Trypanosoma cruzi (known as GP57/51 or cruzipain), an antigen whose serodiagnostic properties were previously established. Cruzipain is selectively adsorbed from crude extracts of $T$. cruzi onto PINC-coated wells; the finding that antibodies bind to epitopes located away from the sites of interaction with r-cystatin or low molecular weight kininogen has allowed for the screening of antibodies in chagasic sera, the methodology being advantageous in that it dispensed prior purification of the proteinase antigen. The PINC-ELISA was then carried out with lysates originating from Leishmania m. amazonensis (amastigotes) or Leishmania donovani (promastigotes). Complexes between solid-phase r-cystatin $\mathrm{C}$ and antigenic ligands in the lysates were again detected. The Leishmania molecules
\end{abstract}

Abbreviations: PINC, proteinase inhibitor from the cystatin superfamily; PINC-ELISA, enzyme-linked immunosorbent assay using cystatin-like inhibitors; cruzipain or GP57/51, major cysteine proteinase from $T$. cnuzi; r-cystatin C, recombinant cystatin C; TBS, Tris-buffered saline solution; BSA, bovine serum albumin; PMSF, phenylmethylsulfonylfluoride; E-64, trans-epoxysuccinyl-Lleucilamido (4-guanidino) butane; CBZ-Phe-Arg-MCA, $N$-carbobenzoxy-phenylalanine, arginine, 7-amidomethyl coumarin.

* Corresponding author. Fax: 55-21-2808193. 
which bound to r-cystatin $\mathrm{C}$, were respectively recognized by serum antibodies from mice chronically infected with $L$. amazonensis or from patients with visceral leishmaniasis. Direct evidence for the presence of cysteine proteinases in lysates from $L$. donovani was then obtained, using synthetic fluorogenic substrates. Due to the broad inhibition profile of r-cystatin $\mathrm{C}$ and the marked antigenicity of parasitic cystcinc protcinases, such enzymes can be readily detected by PINC-ELISA, without requirement for prior knowledge of their substrate specificities.

Keywords: Cysteine proteinase; Cystatin; Kininogen; Immunoassay; Trypanosoma cruzi; Leishmania

\section{Introduction}

Lysosomal cysteine proteinases (cathepsin B, L, $\mathrm{H}$ and S) are members of a widely distributed superfamily of protcins that arc evolutionarily related to papain and are largely responsible for protein turnover in mammalian cells (Barrett and Kirschke, 1981). In pathological states such as sepsis, trauma and cancer, these proteinases have also been found in extracellular fluids (Poole, 1973; Sloane and Honn, 1984; Burleigh et al., 1974; Delaisse et al., 1984), and may thus possibly contribute to tissue destruction in inflammatory reactions. Among the natural inhibitors that arc capable of controlling the extracellular activity of proteinases, only those that belong to the cystatin superfamily are selective for cysteine proteinases (Barrett, 1981; Rawlings and Barrett, 1990). As opposed to $\alpha_{2}$ macroglobulin, which inhibits various subclasses of endopeptidases in an irreversible fashion, the cystatins bind reversibly to just papain-like cysteinyl proteinases (Abrahamson et al., 1987). Again in contrast to $\alpha_{2}$ macroglobulin, the inactivation of the reactive cysteine in the catalytic site of cysteine proteinases does not prevent the formation of tight complexes with cystatins (Poole, 1973). X ray crystal structure analysis of papain complexes with chicken cystatin and human cystatin B (stefin) indicate that the inhibitor's binding to cysteine proteinase active sites is achieved by a wedgeshaped binding region formed by spacially discontiguous regions (Bode et al., 1988; Stubbs et al., $1990)$, together with a second binding region corresponding to the amino-terminal segments of cystatins (Hall et al., 1992). The amino-terminal binding region of the cystatins reaches the specificity pocket of cysteine proteinases, but is still too far from the reactive site cysteine to be attacked.

Two of the nine cystatins known to date are present mainly intracellularly (cystatins, or stefins, $\mathrm{A}$ and $\mathrm{B}$ ), but the remaining are extracellular (cystatins C, D, SN and SA, low molecular weight and high molecular weight kininogens). Except for the kininogens, all cystatins are single-chain proteins of low molecular weight $\left(M_{\mathrm{r}} 12-15000\right)$. Cystatin $\mathrm{C}$ is the more generally distributed of these, being present in all body fluids (Abrahamson et al., 1986). Cystatin C is also the protein with the broadest inhibition profile, binding all cysteine proteinases studied with $K_{\mathrm{i}}<10^{-9}$. The intravascular kininogens are more complex proteins, and contain two functional cystatin domains (Salvesen et al., 1986). They are distributed primarily in blood and synovial fluid (Abrahamson et al., 1986).

Papain-like parasitic cysteine proteinases can be readily inhibited by the cystatins, as exemplified by tight complexes reported for chicken cystatin-histolysin from Entamoeba histolytica $\left(K_{\mathrm{i}} 8\right.$ pM) (Luaces and Barret, 1988) and human cystatin C-cruzipain ( $K_{\mathrm{i}} 60 \mathrm{pM}$; Abrahamson and Scharfstein, unpublished). Of relevance to the present study, the $T$. cruzi cysteine proteinase (GP57/51) (Murta et al., 1990) is a major antigen in chagasic infection (Scharfstein et al., 1986; Arnholdt and Scharfstein, 1991; Gazzinelli et al., 1990; Arnholdt et al., 1993). Also known as cruzipain (Cazzulo et al., 1990) or cruzain (Eakin et al., 1992), this proteinase adds to the growing list of papain-like antigens that elicit potent immunological responses from hosts exposed to parasites (Authié et al., 1992) or to dust mite allergens (Chua et al., 1988). In the present study, we describe a capture immunoassay (PINC-ELISA) 
that explores the binding selectivity of cysteine proteinase interactions with inhibitors from the cystatin superfamily (PINC). The assay can be a general tool in the identification of papain-like antigens in biological fluids.

\section{Materials and methods}

\subsection{Source of cysteine proteinase antigens}

GP57/51 antigen, obtained in enzymatically active form, was purified from crude soluble extracts of $T$. cruzi epimastigotes, as previously described (Lima et al., 1992). In some assays, we used crude soluble extracts, or a partially purified sample obtained by precipitation of crude extracts with $60 \%$ saturated ammonium sulfate (SAS-60). Crude lysates from L. amazonensis were derived from amastigotes collected from lesions of infected BALB/c mice; the parasites were solubilized in $1 \%$ Triton $\mathrm{X}-100$ in $20 \mathrm{mM}$ acetate buffer, pH 5.0, containing EDTA $2 \mathrm{mM}$. Aliquots of phenylmethylsulfonylfluoride (PMSF), at final concentration of $1 \mathrm{mM}$, were added to the lysate, immediately after sonication. After centrifugation ( $18000 \mathrm{rpm}, 20 \mathrm{~min})$, the lysate was aliquoted and stored at $-20^{\circ} \mathrm{C}$. Cell lysates from $L$. donovani (strain LD-1S/MHOM/SD/ 00 -strain $1 \mathrm{~s}$ ) were obtained from stationary phase (7 days) promastigotes grown in BHI-supplemented with $10 \%$ of fetal calf serum and $N^{\prime}, N^{\prime}, N^{\prime}$-based medium, containing $2 \%$ agar and $5 \%$ fibrinated rabbit blood. The cell pellet $\left(10^{9}\right.$ cells) recovered after $11 \mathrm{~s}$ of microcentrifugation was washed three times with ice-cold PBS and were resuspended in $1 \mathrm{ml}$ of a buffer containing $10 \mathrm{mM}$ Tris $\mathrm{HCl}, 2 \mathrm{mM}$ EDTA, supplemented with $2 \mathrm{mM}$ PMSF and $1 \mu \mathrm{g} / \mathrm{ml}$ of pepstatin (Sigma Chemical Company, St. Louis, MO). The suspension was subjected to freeze and thaw $(5 \times$ 3 min cycles), and centrifuged to separate the soluble fraction. The insoluble debris was solubilized ( $1 \mathrm{~h}$ in ice) with $1 \%$ of Triton X-100 buffered with $10 \mathrm{mM}$ Tris- $\mathrm{HCl}, 25 \mathrm{mM} \mathrm{NaCl}, 2 \mathrm{mM}$ EDTA, supplemented with $1 \mathrm{mM}$ PMSF. The lysate was cleared out of aggregates by centrifugation and stored as already described. Protein concentra- tions of $T$. cruzi soluble proteins and of Triton $X-100$ containing lysates were determined by the Bradford dye-binding assay (Bradford, 1976) and by the DC-Protein assay (Bio-Rad, Richmond, CA) performed according to the manufacturer's instructions, respectively. Endopeptidase activity of cysteine proteinases was monitored by spectrofluorometry, using CBZ-Phe-Arg-MCA as fluorogenic substrate, as previously described (Lima et al., 1992). PMSF, E-64, pepstatin, leupeptin and $o$-phenanthroline were obtained from Sigma, St. Louis.

\subsection{Capture reactants}

Human r-cystatin $C$, identical to the native protein in structure and functional properties, was obtained in an $E$. coli expression system as earlier described (Abrahamson et al., 1988). Human low molecular weight kininogen, isolated from blood according to Johnson et al. (1987), was a gift from J.A. Guimarāes (UFRJ). Bovine serum albumin (BSA), used as control, was obtaincd from Sigma.

\subsection{Antibodies}

Mouse monoclonal antibodies to GP57/51 (cruzipain) were obtained by established methods (Köhler and Milstein, 1975). Mab 212BH6 is an IgG1 antibody that binds to native cruzipain as well as to denatured r-cruzipain 2 expressed in $E$. coli. Mouse polyclonal antiscra to GP57/51 wcre obtained from BALB/c mice hyperimmunized with purified GP57/51. Serum from chagasic patients was supplied by Dr. Roberto Pedrosa (University Hospital-UFRJ). Sera from patients with visceral leishmaniasis (caused by infection with $L$. chagasi) and non-infected controls from endemic areas of Bahia were obtained at the facilities of the Serviço de Imunologia Edgar Santos, UFBa. Sera from non-endemic areas derived from laboratory staff.

\subsection{PINC-ELISA}

Plating of wells $(100 \mu 1)$ was carried out overnight, with r-cystatin $\mathrm{C}$, low molecular weight 
kininogen or BSA diluted in TBS ( $\mathrm{pH} 7.2)$, at the indicated concentrations. The wells were blocked (90 min, room temperature) with TBS$0.5 \%$ Tween. After four washing cycles of $5 \mathrm{~min}$

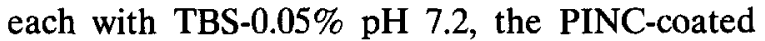
wells were incubated ( $2 \mathrm{~h}$, room temperature) with cell lysates or with purified $n$-cruzipain solutions, diluted as required in TBS-0.05\% Tween (pH 7.2). Some of the PINC-coated wells were treated with buffer alone (TBS-0.05\% Tween) containing the same final content of cell lysis buffer. After removing the parasite products from the wells, these were treated ( $1 \mathrm{~h}$, room temperature) with antibodies diluted $1 / 100$ (human or mouse serum) or $1 / 1000$ (monoclonal antibodies) in TBS- $0.05 \%$ Tween. The wells were washed three times as described above, and primary antibodies bound to wells were quantified following reaction with peroxidase immunoconjugates (Sigma Chem. Co., St. Louis), diluted 1:1500 in TBS- $0.05 \%$ Tween, and $o$-phenylenediamine as the substrate for color development. In some experiments, we used alkaline phosphatase alkaline anti-human or mouse $\mathrm{IgG}$ diluted $1 / 1500$ in

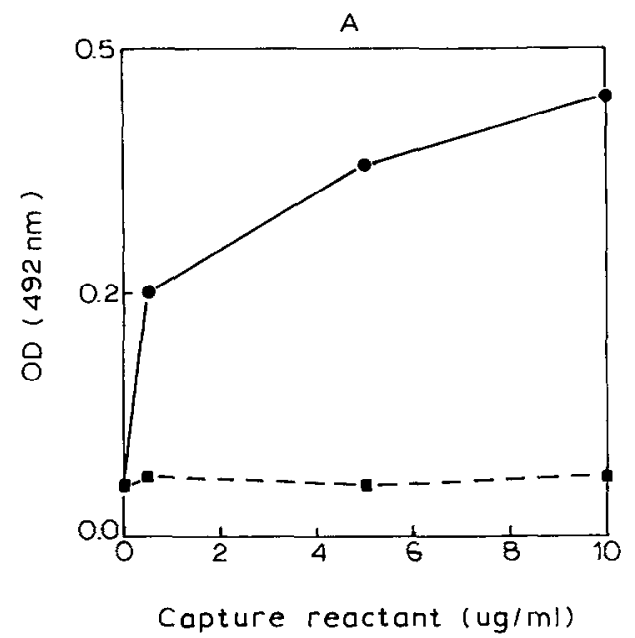

the same buffer, and $p$-nitrophenyl phosphate as the substrate (all from Bio-Rad). Readings of optical density were done at 492 and $405 \mathrm{~nm}$, respectively. Assays were always carried in duplicates $(\mathrm{SD}<10 \%)$. The data shown in the figures are representative of two independent experiments.

\section{Results}

The efficacy of r-cystatin $\mathrm{C}$ as a capture reactant was initially evaluated using excess of purified GP57/51 $(20 \mu \mathrm{g} / \mathrm{ml})$ as a source of soluble proteinase, and Chagas' discase scrum or Mab 212BH6 as the antibody source. The capture of purified cruzipain was dose-dependent (not shown). Crude soluble extracts were then used with the same intent (Fig. 1A). The data obtained with chagasic serum (normal human serum as controls) show that the reaction reached plateau levels at $10 \mu \mathrm{g} / \mathrm{ml}$ of cystatin C. BSA, assayed at the same concentration range, was unable to capture the antigen. The experiments shown in Fig.

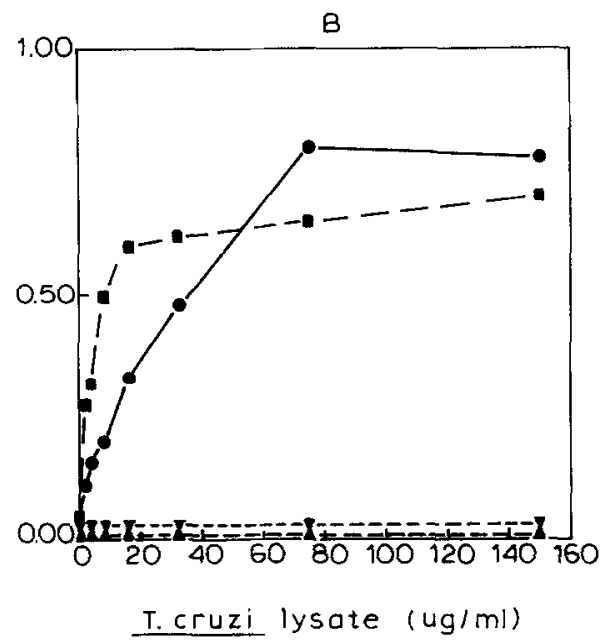

Fig. 1. A: PINC-ELISA, dose dependence of r-cystatin C. Increasing quantities of antigenic cysteine proteinases were captured from $T$. cruzi lysates when r-cystatin C (circles) was used as the capture reactant. Solid-phase BSA (squares), used in the same concentration range, did not capture the parasite proteinase. Chagasic sera were employed to quantify $T$. cruzi immunoreactive products bound to cystatin $C$. When exposed to normal human sera, the cystatin $C$-bound cruzipain antigen did not show any reactivity. $B$ : dose dependence of $T$. cruzi lysate. A fixed concentration $(10 \mu \mathrm{g} / \mathrm{ml})$ of r-cystatin C (circles), or low molecular weight kininogen (squares) was plated to wells and these were used to characterize the dose-response dependency of $T$. cruzi lysates. Specificity controls: chagasic serum reactivity with cystatin C-coated wells not exposed to $T$. cruzi extracts (triangles) and normal human serum (inverted triangles) exposed to cystatin $C$-binding antigens were all tested at 1:100 dilution. 
1B indicate that low molecular weight kininogen could be also used as an alternative capture reactant. Comparison of the curves obtained for these two PINC reveals a steeper slope for the bivalent kininogen, at the low concentration range of the cruzipain-rich extract. The specificity of the serological reactions was demonstrated in the experiment shown in Fig. 1B. As expected, in the absence of a potential source of protease, chagasic serum fails to react with wells coated with $r$ cystatin $\mathrm{C}$ or with low molecular weight kininogen. Moreover, normal human serum did not react with the PINC-captured antigen. As already mentioned, the reaction with Mab 212BH6 confirmed the identity of the r-cystatin-bound antigen as the major cysteine protease of $T$. cruzi (Fig. 3). As shown in Fig. 2 (inset), the reactivity of chagasic serum as measured by PINC-ELISA correlated with those obtained by conventional ELISA (using purified $n$-cruzipain). To evaluate if the proteinase was the only cystatin-binding antigen contained in extracts from epimastigotes, the sample was loaded on cystatin C-Sepharose columns. After subjecting the column to extensive washings with PBS-EDTA $2 \mathrm{mM}, 0.05 \%$ Tween,

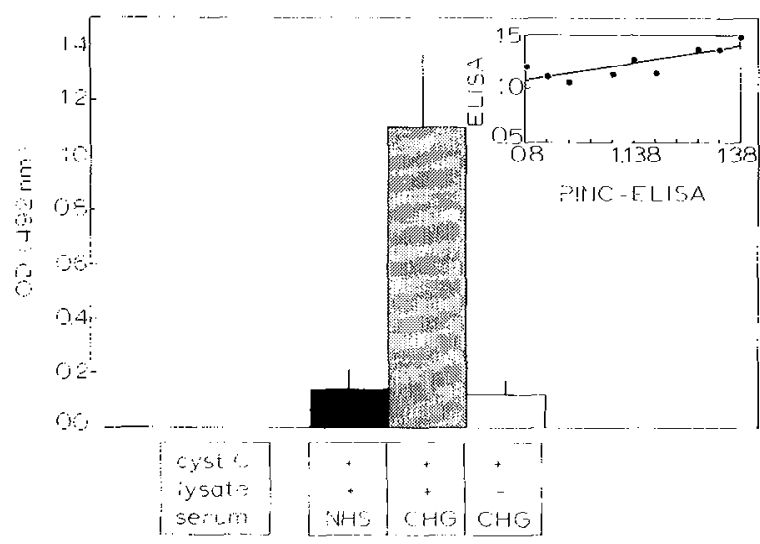

Fig. 2. Screening of anti-cruzipain antibodies in chagasic sera $T$. cruzi antigens (cruzipain) adsorbed onto $r$-cystatin C-coated wells were used to screen serum samples $(1: 100)$ from Chagas' disease patients $(n=15)$ or normal controls $(n=12)$. Wells containing r-cystatin $\mathrm{C}$ alone, and subsequently treated with chagasic serum were used as specificity controls. Surfaces coated with BSA and exposed to $T$. cruzi lysate did not yield reactions with chagasic sera (not shown). Inset: correlation of the reactivity pattern of some of the chagasic serum samples tested by PINC-ELISA or by conventional ELISA.

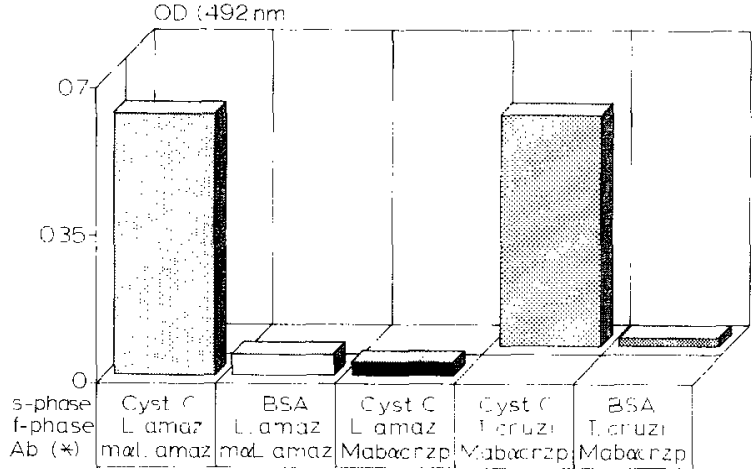

Fig. 3. Idcntification of cystatin C-binding antigens in Leishmania amazonensis. Lysates from freshly obtained amastigotes ( $L$. amazonensis) or epimastigotes $(T$. cruzi) were added to wells previously coated with $10 \mu \mathrm{g} / \mathrm{ml}$ of r-cystatin C or BSA. Immune serum, tested at 1:100, was derived from $B A L B / c$ mice chronically infected with $L$. amazonensis. BALB $/ \mathrm{c}$ normal serum did not react with antigens captured from $L$ amazonensis or $T$. cruzi lysates. Reactions with monoclonal antibodies to GP57/51 (cruzipain) allowed its identification as the cystatin $\mathrm{C}$-binding antigen captured from crude lysates of $T$. cruzi.

the ligands were eluted with $8 \mathrm{M}$ and were characterized after dialysis. Enzymatically active cruzipain was partially recovered, and purity was confirmed by silver stained SDS-PAGE gels, and immunoblotting with Mab 212BH6 (data not shown).

We then sought to identify antigenic cysteine proteinases in lysates from other species of pathogenic trypanosomatids. Serum from mice chronically infected with $L$. amazonensis was used to investigate whether lysates from amastigotes contained such molecules. The results shown in Fig. 3 indicate that it contained cystatin-binding antigens. Normal mouse serum did not react with the antigen (not shown). Non-specific adsorption of $L$. amazonensis antigens to the wells was ruled out, because the reaction with the immune serum did not develop when r-cystatin was substituted by BSA. Morcover, the L. amazonensis antigen(s) bound to r-cystatin $\mathrm{C}$ did not react with a monoclonal antibody directed against the polypeptide portion of the $T$. cruzi enzyme (GP57/51). Similar data were obtained when we screened a small number of serum samples obtained from patients with visceral leishmaniasis $(n=8)$, as part of an 
initial attempt to identify cysteine proteinase antigens in cell lysates prepared from $L$. donovani promastigotes. Six of these serum showed positive reactions when tested by PINC-ELISA (Fig. 4A). Similarly to the assays described for $T$. cruzi extracts, the Kalazar sera did not react directly with the r-cystatin $\mathrm{C}$, nor did we observe reactions of normal human serum with the $L$. donovani molecules bound to r-cystatin $\mathrm{C}$ (Figs. 4A and 4B). Again, the antibody reaction was dependent on the concentration of the lysate (Fig. 4B). However, we detected some reactivity in one of the sera (1 out of 10, not shown) originating from the geographic were visceral leishmaniasis is endemic, thus suggesting that this particular individual might have had asymptomatic disease. Collectively, these experiments suggested that the lysates from $L$. donovani pro- mastigotes contained matured forms of cysteine proteinases. This possibility was subsequently confirmed when we assayed the protease activity with a synthetic fluorogenic substrate, CBA-PheArg-MCA. Measurements of the initial rate of hydrolysis (Fig. 5) indicated that these lysates indeed contained an endopeptidase activity that was markedly sensitive to inhibition by E-64 and $r$-cystatin $\mathrm{C}$, but not by aprotinin. A discrete inhibition was observed when the samples treated with pepstatin $(9 \%$, not shown) and $o$ phenanthroline. The molecular characterization of the cystatin C-binding antigen of $L$. donovani promastigotes is under progress. Preparative scale analysis of the ligands bound to cystatin C-affinity columns has revealed the presence of a single polypeptide of $69 \mathrm{kDa}$ in efluents from $8 \mathrm{M}$ urea. When tested with Kalazar serum $(n=4)$ by con-
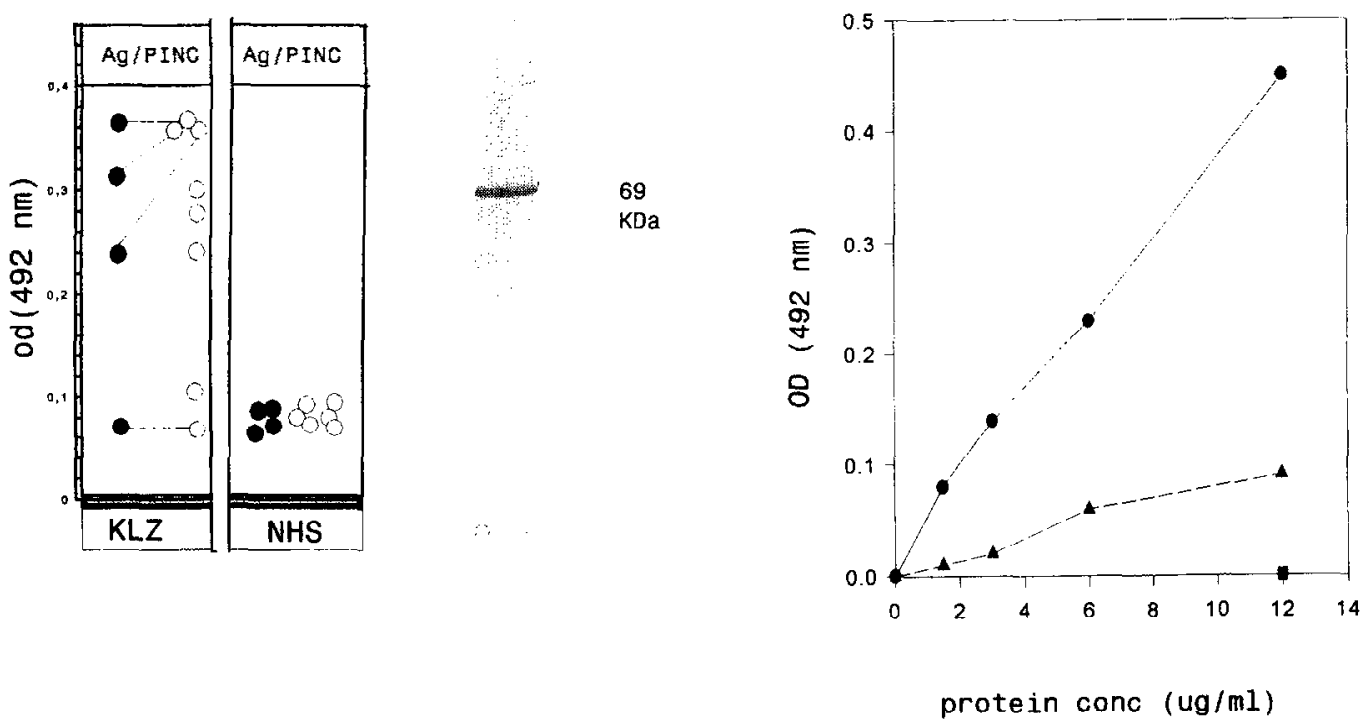

Fig. 4. $A$ : cystatin C-binding proteins are antigenic in visceral leishmaniasis. Individual serum samples from patients with Kalazar (and normal human serum, as controls) assayed by PINC-ELISA (open circles) or by conventional ELISA with affinity-purified Ld69 antigen (closed circles). Purity of Ld69 is shown at the right side of the figure (SDS-PAGE, denaturing conditions/reduced sample, silver stained gel). $B$ : dose response. Increasing concentrations of lysates from $L$. donovani promastigotes were incubated with solid-phase r-cystatin C. Antigen captured by this PINC was quantified following incubation with pooled Kalazar sera, at 1:100 (closed circles). Pooled normal sera were used as specificity control (black squares). Serum from an individual without clinical history of Kalazar (but from the same endemic area) is represented by closed triangles. 


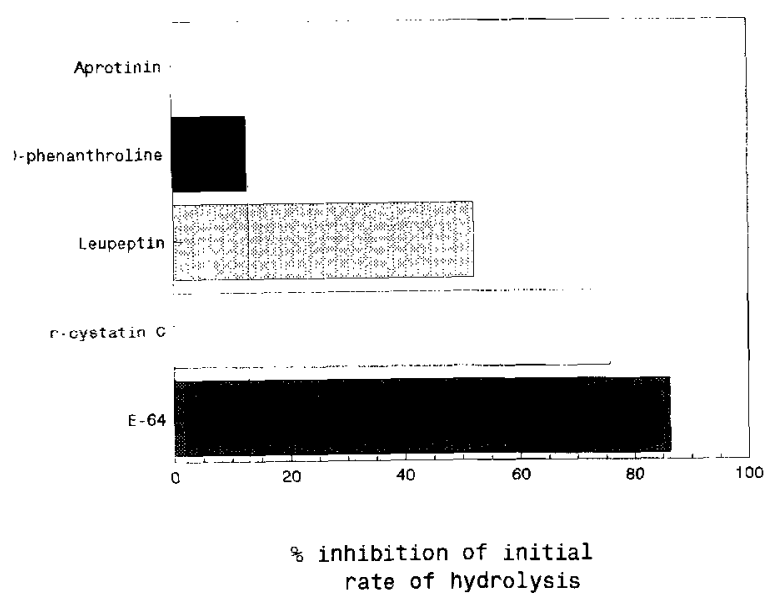

Fig. 5. Evidence for the presence of active cysteine proteinases in lysates from $L$. donovani promastigotes. Cell lysates at $60 \mu \mathrm{g} / \mathrm{ml}$ protein concentration were diluted $1 / 4$ in an activation buffer containing phosphate-citrate $10 \mathrm{mM}, \mathrm{NaCl}$ $150 \mathrm{mM}$, EDTA $2 \mathrm{mM}$, pH 5.5 and supplemented with DTT 5 $\mathrm{mM}$ for $1 \mathrm{~h}$, on ice. Except for the control tubes, the samples were supplemented with the test inhibitors (E-64, $20 \mu \mathrm{M}$; r-cystatin C $10 \mu \mathrm{g} / \mathrm{ml}$; pepstatin, $5 \mu \mathrm{M}$; aprotinin, $2 \mu \mathrm{g} / \mathrm{ml}$; leupeptin, $2 \mu \mathrm{M} ; o$-phenanthroline, $500 \mu \mathrm{M}$. Values for initial rates were obtained using CBZ-Phe-Arg-MCA at 10 $\mu \mathrm{M}$ final concentration, in the same buffer described above, supplemented with 5\% DMSO. Percent inhibition of initial rates were calculated with reference to controls (enzyme samples containing appropriate volume of solvent used for inhibitor preparation).

ventional ELISA, purified Ld69 displayed reactions in values that were comparable to those obtained by PINC-ELISA, suggesting that this protein may be the dominant, if not exclusive, cystatin C-binding antigen of $L$. donovani promastigotes.

\section{Discussion}

The activities of papain-like cysteine proteinases can be inhibited by proteins belonging to the cystatin superfamily. Present in human plasma and tissue fluids, the kininogens and cystatin $C$ are respectively thought to regulate the potentially damaging action of extracellular cysteine proteinases. Secretion of the latter was reported to occur in pulmonary, periodontal and joint dis- eases, and in cancer states (Brocklehurst et al., 1987).

Here we show that specificity properties of the cystatins can be explored to identify antigenic cysteine proteinases. This may be particularly useful to research on immunoparasitology and allergy, due to the growing number of reports associating immune responses to papain-like proteinases to human and veterinary disease (Arnholdt and Scharfstein, 1991; Arnholdt et al., 1993; Authié et al., 1992; Chua et al., 1988). Analysis of the crystal structure of a few members of the superfamily, and sequence alignment of many others cysteine proteinases suggests that structural variability is higher at solvent-exposed regions of these enzymes. This is in contrast to the relatively conserved residues forming the active site cleft, such as those involved in the complimentary interactions with the wedge-shaped edge of cystatin inhibitors. Thus, a differential distribution of antibody and cystatin binding sites could be predicted, and if true, should allow for the formation of ternary complexes with cysteine proteinases. We then sought to investigate if these interactions could have practical value for the diagnosis of infections caused by trypanosomatids. PINC-ELISA was at first optimized with GP57/51 (cruzipain), the major cysteine proteinase from $T$. cruzi. Cell lysates containing mature forms of this lysosomal proteinase were exposed to microtiter wells coated with $\mathrm{r}$-cystatin $\mathrm{C}$ or low molecular weight kininogen; both were of human origin, to preclude reactivity with natural antibodies in human serum. At low concentrations of the soluble extract, the bivalent kininogen bound more cruzipain than the monovalent $\mathrm{r}$-cystatin $\mathrm{C}$, probably reflecting differences in their avidity for the proteinase. The antigenicity of cruzipain bound to r-cystatin $C$ was evidenced with $\mathrm{mAb}$ to the enzyme or with chagasic serum. Previous studies suggested that human antibodies bind to epitopes preferentially localized at the C-terminal portion of the proteinase (Scharfstein et al., 1985; Arnholdt and Scharfstein, 1991; Martinez et al., 1993). The antibody specificities stimulated during human infection appear to be primarily directed against epitopes that are distant from the active site. This is also suggested by the 
results obtained when we plated $n$-cruzipain directly on the plastic wells, since the binding of anti-cruzipain IgG (purified from the sera of several individual patients) did not interfere with the enzyme's subsequent interaction with soluble $r-$ cystatin C (data not shown). Consistent with these findings, the binding sites for PINC, either cystatin $\mathrm{C}$ or low molecular weight kininogen, did not hinder the access of Mab 212BH6 to the epitope, mapped to the C-terminal domain of cruzipain (unpublished observations). Based on the biochemical analysis of the r-cystatin C-binding antigens recovered by means of affinity chromatography (not shown), we have concluded that cruzipain is the only $T$. cruzi antigen recognized by PINC-ELISA.

We then used PINC-ELISA to identify antigenically active cysteine proteinases from different species of Leishmania. To approach this problem in the context of cutaneous leishmaniasis, immune serum from mice chronically infected with $L$. amazonensis was used as a potential antibody source. The assay promptly led to the detection of PINC-binding antigens in lysates from amastigotes. Taking the lead from the immunoassay data, we were able to detect (data not shown) E-64 sensitive proteinase activities in the lysate from $L$. amazonensis amastigotes (North et al., 1990). It is noteworthy that the L. amazonensis molecules bound to cystatin $\mathrm{C}$ does not share the cruzipain epitope defined by Mab 212BH6; this is not unexpected, considering the sequence differences reported for some of these cysteine proteinases (Eakin et al., 1990; Traub-Czeko et al., 1993).

PINC-ELISA was then applied to detect cystatin-binding antigens associated with visceral leishmaniasis (Kalazar), a systemic form of disease which, in north Brazil, is caused by infection with $L$. chagasi. Kalazar is associated with marked depression of cellular immunity to leishmanial antigens (Carvalho et al., 1988) and with polyclonal B cell responses (Ghose et al., 1980). Lysates obtained from $L$. donovani promastigotes were chosen as a potential source of the proteinase, and immune sera from the Kalazar patients (as compared to sera from non-infected controls from the same endemic area) were as- sayed. We found out that most sera $(75 \%)$ indeed contained specific antibodies to cystatin-bound promastigote antigens. The $L$. donovani antigen also reacted with chronic chagasic sera (not shown), thus may not be adequate for diagnostics tests. The structural basis of this cross-reactivity remains to be determined. As previously discussed, the cystatin $\mathrm{C}$-binding antigens from the $L$. donovani likely consist of mature forms of cysteine proteinase; this conclusion is supported by the finding that $L$. donovani lysates indeed contained an endopeptidase that was inhibited by E-64 or cystatin C, as would be expected from papain-like proteinases. Preliminary characterization of the cystatin-binding antigens was carried out by affinity chromatography; a single band of $69 \mathrm{KDa}$ was recovered from $8 \mathrm{M}$ urea column efluents, but the endopeptidase activity was markedly impaired, probably due to denaturation. Sequence analysis is under progress to confirm that the protein is indeed related to the papain superfamily. Upon evaluation by conventional ELISA, the purified Ld69 antigen yielded a similar pattern of reactivity with Kalazar serum, as compared to those obtained by PINC-ELISA. Because it dispenses a prior knowledge of the enzyme's substrate specificity, PINC-ELISA may prove valuable as a general screening tool for the detection of antigenic cysteine proteinase in parasitic as well as in allergic diseases.

\section{Acknowledgements}

Supported by grants from the European Community (EEC), FINEP, CNPq, and CEPG-UFRJ. The technical assistance of João Marreiro Sobrinho, Alda M. Alves, Alexandre Silveira and Cristiane Domingues is acknowledged.

\section{References}

Abrahamson, M., Salvesen, G., Barrett, A.J. and Grubb, A. (1986) Isolation of six cysteine proteinase inhibitors from human urine. Their physicochemical and enzyme kinetic properties and concentrations in biological fluids. J. Biol. Chem. 261, 11282. 
Abrahamson, M., Ritonja, A., Brown, M.A., Grubb, A. Machleidt, W. and Barrett, A.J. (1987) Identification of the probable inhibitory reactive sites of the cysteine proteinase inhibitors human cystatin $\mathrm{C}$ and chicken cystatin. J. Biol. Chem. 262, 9688.

Abrahamson, M., Dalboge, H., Olafson, I., Carlsen, S. and Grubb, A. (1988) Efficient production of native, biologically active human cystatin $\mathrm{C}$ by Escherichia coli. FEBS Lett. 236, 14.

Arnholdt, A.C.V. and Scharfstein, J. (1991) Imunogenicity of Trypanosoma cruzi cysteine proteinase. Res. Immunol. 142, 146.

Arnholdt, A.C.V., Piuvezam, M.R., Russo, D.M., Lima, A.P.C.A., Pedrosa, R., Reed, S.G. and Scharfstein, J. (1993) Analysis and partial èpitope mapping of human $T$ cell responses to Trypanosoma cruzi cysteinyl proteinases. J. Immunol. 151, 3171.

Authié, E., Muteti, D.K., Mbawa, Z.R., Lonsdale-Eccles, J.D., Webster, P. and Wells, C.W. (1992) Identification of a 33 kilodalton immunodominant of Trypanosoma congolense as a cysteine protease. Mol. Biochem. Parasitol. 56, 103.

Barrett, A.J. (1981) Cystatin, the egg white inhibitor of cysteine proteinases. Methods Enzymol. 80, 771-778.

Barrett, A.J. and Kirschke, H. (1981) Cathepsin B, cathepsin H, cathepsin L. Methods Enzymol. 80, 535.

Bradford, M.M. (1976) A rapid and sensitive method for the Quantitation of microgram quantities of protein utilizing the principle of protein-dye binding. Anal. Biochem. 72, 248.

Bode, W., Engh, R., Musil, D., Thiele, U., Huber, R., Karshikov, A., Brzin, J., Kos, J. and Turk, V. (1988) The $2.0 \AA \mathrm{X}$-ray crystal structure of chicken egg white cystatin and its possible mode of interaction with cysteine proteinases. EMBO J. 7, 2593.

Brocklehurst, K., Willenbrock, F. and Salih, E. (1987) Cysteine proteinases. In: A. Neuberger and K. Brocklehurst (Eds.), Hydrolytic Enzymes. Elsevier, Amsterdam, p. 39.

Burleigh, M.C., Barrett, A.J. and Lazarus, G.S (1974) Cathepsin B1: A lysosomal enzyme that degrades native collagen. Biochern. J. 137, 387.

Carvalho, E.M., Bacellar, O.A., Reed, S.R., Barral, A. and Rocha, H. (1988) Visceral leishmaniasis: a disease associated with inability of lymphocytes to activate macrophages to kill leishmania. Braz. J. Med. Biol. Res. 21, 85.

Cazzulo, J.J., Franke-Cazzulo, M.C., Martinez, J. and Franke de Cazzulo, B.M.F. (1990) Some kinetic properties of a cysteine proteinase (cruzipain) from Trypanosoma cruzi. Biochim. Biophys. Acta 1037, 186.

Chua, K.Y., Stewart, G.A., Thomas, W.R., Simpson, R.J., Dilworth, R.J., Ploza, T.M. and Turner, K.J. (1988) Sequence analysis of cDNA coding for a major house dust mite allergen, Derp 1. Homology with cysteine proteases. J. Exp. Med. 167, 175.

Delaisse, J.-M., Eeckhout, Y. and Vaes, G. (1984) In vivo and in vitro evidence for the involvement of cysteine proteinases in bone resorption. Biochem. Biophys. Res. Commun. 125,441 .
Eakin, A.E., Bouvier, J., Sakanari, J.A., Craik, C.S., and McKerrow, J.H. (1990) Amplification and sequencing of genomic DNA fragments encoding cysteine proteinases from protozoan parasites. Mol. Biochem. Parasitol. 39, 1.

Eakin, A.E., Mills, A.A., Harth, G., McKerrow, J.H. and Craik, C.S. (1992) The sequence, organization and expression of the major cysteine protease (cruzain) from $T y$ panosoma cruzi. J. Biol. Chem. 267, 7411.

Gazzinelli, R.T., Leme, V.C.M., Cançado, J.R., Gazzinelli, G. and Scharfstein, J. (1990) Identification and partial characterization of $T$. cruzi antigens recognized by $T$ cells and immune sera from chagasic patients. Infect. Immun. 58, 1437.

Ghose, A.C., Haldar, J. P., Pal, S.C., Mishra, B.P. and Mishra K.K. (1980) Serological investigations on Indian Kala-Azar. Clin. Exp. Immunol. 40, 318.

Hall, A., Abrahamson, M., Grubb, A., Trojnar, J., Kania, P., Kasprzykowska, R. and Kasprzykowski, F. (1992) Cystatin $\mathrm{C}$ based peptidyl diazomethanes as cysteine proteinase inhibitors: Influence of the peptidyl chain lenght. J. Enzyme Inhib. 6, 113.

Johnson, D.A., Salvesen, G., Brown, M.A. and Barrett, A.J. (1987) Rapid isolation of human kininogens. Thromb. Res. $48,187$.

Köhler, G. and Milstein, C. (1975) Continuous cultures of fused cells secreting antibody of predefined specificity. Nature 256, 495.

Lima, A.P.C.A., Scharfstein, J., Storer, A.C. and Menard, R (1992) Temperature-dependent substrate inhibition of the cysteine proteinase (GP57/51) from Trypanosoma cruzi. Mol. Biochem. Parasitol. 56, 355.

Luaces, A.L. and Barret, A.J. (1988) Affinity purification and biochemical characterization of hystolysin, the major cysteine proteinase of Entamoeba histolytica. Biochem. J. $250,903$.

Martinez, J., Campetella, O., Frasch, A.C. and Cazzullo, J.J. (1993) The reactivity of sera from chagasic patients against different fragments of cruzipain, the major cysteine proteinase from Trypanosoma cruzi, suggest the presence of defined antigenic and catalytic domains. Immunol. Lett. 35, 191.

Murta, A.C.M., Persechini, P.M., Souto-Padrón, T., De Souza. W., Guimarães, J.A. and Scharfstein. J. (1990) Structural and functional identification of GP57/51 antigen of Try. panosoma cruzi as a cysteine proteinase. Mol. Biochem. Parasitol. 43, 27.

North, M.J., Mottram, J.C. and Coombs, G.H. (1990) Cysteine proteinases of parasitic Protozoa. Parasitol. Today 6, 270

Poole, A.R. (1973) Tumor lysosomal enzymes and invasive growth. In: J. T. Dingle (Ed.), Lysosomes in Biology and Pathology, Vol. 3. North-Holland Publishing Co., Amsterdam, p. 303.

Rawlings, N.D. and Barrett, A.J. (1990) Evolution of proteins of the cystatin superfamily. J. Mol. Evol. 30, 60.

Salvesen, G., Parkes, C., Abrahamson, M., Grubb, A. and Barrett. A.J. (1986) Human low molecular weight kininogen contains three copies of a cystatin sequence that are 
divergent in structure and in inhibitory activity for cysteine proteinases. Biochem. J. 234, 429.

Scharfstein, J., Luquetti, A.O., Murta, A.C.M., Senna, M., Rassi, A., Rezende, J.M. and Mendonça-Previato, L. (1985) Chagas' disease: serodiagnosis with purified Gp-25. Am. J. Trop. Med. Hyg. 34, 1153.

Scharfstein, J., Schechter, M., Senna, M., Peralta, J.M., Mendonça-Previato, L. and Miles, M.A. (1986) Trypanosoma cruzi: characterization and isolation of a $57 / 51000$ molecular weight surface glycoprotein (GP-57/51) expressed by epimastigotes and bloodstream trypomatigotes. J. Immunol. 137, 133 ..
Sloane, B.F. and Honn, K.V. (1984) Cysteine proteinase and metastasis. Cancer Met. Rev. 3, 249.

Stubbs, M.T., Laber, B., Bode, W., Huber, R., Jeralka, R., Lenarcic, B. and Turk, V. (1990) The refined 2.4 A X-ray structure of recombinant human stefin $B$ in complex with the cysteine proteinase papain: a novel type of proteinase inhibitor interaction. EMBO J. 9, 1939.

Traub-Czeko, Y.M., Duboise, S.M., Boukai, and McMahonPratt, D. (1993) Identification of two distinct cysteine proteinase genes of Leishmania pyfanoi axenic amastigotes. Mol. Biochem. Parasitol. 57, 101. 